\title{
Editorial
}

\section{Diagnosis of Rheumatic Fever and Rheumatic Heart Disease in Evolution}

\author{
AKM Monwarul Islam¹, HI Lutfur Rahman Khan²
}

(Bangladesh Heart Journal 2015; 30(1) : 1-4)

Acute rheumatic fever (ARF) and consequent rheumatic heart disease (RHD) are important public health issues in developing countries, and marginalized people of some developed countries. Globally, RHD remains the most-common cardiovascular disease (CVD) in young people aged $<25$ years. ${ }^{1}$ The overall incidence of ARF varies from 5 to 51 per 100,000 population with a mean of 19 per $100,000^{2}$, while the global burden of RHD in the 5-14 year old children has been estimated to be 0.8 - 5.7 per 1000 with a median of 1.3 per $1000^{3,4}$. With increased public awareness, improvement in socioeconomic conditions, availability of effective antibiotics and possible changes in bacterial pathogenicity, clinically manifest cases of ARF became uncommon in many parts of the world, including Bangladesh. However, subclinical cases of RF continue to occur, as evidenced by prevalence of chronic RHD.

ARF has got no definite test to diagnose, and the symptoms and signs of the disease are shared by other infective and inflammatory conditions. So, historically there was significant heterogeneity in diagnostic approach to the disease, which, in the language of $\mathrm{T}$. Jones in 1944, 'remains one of the important soluble medical problems of our day'. ${ }^{5}$ Being requested by the Subcommittee on Cardiovascular Diseases of the National Research Council of USA for reiteration of the diagnostic criteria of rheumatic fever, a new set of criteria was formulated by $\mathrm{T}$. Jones in 1944, which was later well-accepted throughout the world as the Jones criteria, for diagnosis of ARF. ${ }^{5}$ Over the subsequent decades, these Jones criteria underwent periodic modifications and updates, including the last modification in $1992 .{ }^{6}$ Each change was made to improve the specificity of the criteria at the expense of sensitivity, largely in response to the falling incidence of ARF in the USA. As a result, the

1. Assistant Professor, Department of Cardiology, Jessore Medical College, Jessore. Email: drmonwarbd@yahoo.com

2. Professor, Department of Cardiology, Anwer Khan Modern Medical College, Dhaka. criteria may not be sensitive enough to pick up the disease in high-incidence populations, where the consequences of underdiagnosis may be greater than those of overdiagnosis. Recognizing this issue, World Health Organization (WHO) ${ }^{7}$, and later, Australian ${ }^{8}$ and New Zealand ${ }^{9}$ guidelines set up their own criteria for the diagnosis of ARF as a considerable deviation from the Jones criteria. In this continuum, recently, the American Heart Association (AHA) has published her 'Revision of the Jones Criteria for the Diagnosis of Acute Rheumatic Fever in the Era of Doppler Echocardiography'. ${ }^{10}$ This Revision of the Jones Criteria has, for the first time, set up separate criteria in accordance with the degree of prevalence of ARF/RHD across the world. Low-risk populations have been defined as are those with ARF incidence $<2$ per 100,000 school-aged children or allage rheumatic heart disease prevalence of < 1 per 1000 population per year. On the other hand, children not clearly from a low-risk population have been considered to be at moderate to high risk depending on their reference population. ${ }^{10}$

Like the previous versions, the 'Jones Criteria, 1992 update' includes a number of well-known major and minor criteria. Carditis, arthritis, chorea, subcutaneous nodules and erythema marginatum constitute the major criteria, while minor criteria include fever, arthralgia, raised erythrocyte sedimentation rate (ESR), C-reactive protein (CRP), or leukocytosis, prolonged PR interval on ECG, a previous history of ARF or RHD;diagnosis of first attack of ARF needs presence of either 2 major criteria, or 1 major criterion and 2 minor criteria, along with evidence of preceding group A streptococcal infection. ${ }^{6}$ Classically, carditis has been diagnosed by auscultation of typical murmurs of mitral or aortic valve regurgitation, or both; echocardiographic evidence is not needed. Contrary to this classical approach, current evidence indicate that, a number of cases of carditis, known as 'subclinical carditis', may actually go undetected by cardiac auscultation only, leading to failure of diagnosis of ARF. 
The prevalence of subclinical carditis ranged from 0$53 \%$, with the weighted pooled prevalence $16.8 \%$ in a meta-analysis. ${ }^{11}$ On the other hand, echocardiography, especially the Doppler modality, is much more efficient in this regard. ${ }^{10}$ Therefore, the recent guidelines unequivocally recognize the role of echocardiography in confirmation, follow up and exclusion of the diagnosis of rheumatic carditis. ${ }^{8-10}$ The 2015 Revision of Jones criteria has advocated that echo with Doppler should be performed in all cases of confirmed and suspected ARF (class I; level of evidence B), and that should be performed to assess whether carditis is present in absence of auscultatory findings, particularly in moderate- to highrisk populations and when ARF is considered likely (class I; level of evidence B). ${ }^{10}$

For both low-risk populations, as well as, for moderateand high-risk populations, carditis as a major criterion has been defined as clinical and/or subclinical carditis. Analogous to the Doppler echo diagnostic criteria for RHD by the World Heart Federation (WHF) ${ }^{12}$, AHA, for the first time, has set up criteria for Doppler echocardiographic diagnosis of rheumatic valvulitis. For pathological mitral regurgitation, all 4 criteria should be met: seen in at least 2 views, jet length $>2 \mathrm{~cm}$ in at least 1 view, peak velocity $>3 \mathrm{~m} / \mathrm{s}$, and pan-systolic jet in at least 1 envelope. For pathological aortic regurgitation, again all 4 criteria to be met: seen in at least 2 views, jet length $>1 \mathrm{~cm}$ in at least 1 view, peak velocity $>3 \mathrm{~m} / \mathrm{s}$, and pan-diastolic jet in at least 1 envelope. ${ }^{10}$

Typically, the arthritis of ARF is a migratory polyarthritis, involving the large joints e.g. knees, ankles, elbows, and wrists. Arthralgia has been recognized as a minor criterion. Subsequent observations indicate that, aseptic monoarthritis may also be important as a clinical manifestation of ARF in selected high-risk populations. The 2012 Australian guideline considers aseptic monoarthritis or polyarthralgia as a major manifestation in place of polyarthritis when alternative diagnoses have been carefully excluded. ${ }^{8}$ In the recently published 2015 AHA guideline ${ }^{10}$, monoarthritis has been considered to be part of the ARF spectrum in patients from moderateto high-risk populations (class I; level of evidence C). On the other hand, polyarthralgia has been recognized as a major manifestation only in moderate- or high-incidence populations and only after careful consideration and exclusion of other causes of arthralgia such as autoimmune, viral, or reactive arthropathies (class $\mathrm{llb}$; level of evidence $\mathrm{C}$ ). Monoarthralgia, in this population, has been a minor manifestation.
In the 2015 Revision of Jones Criteria, fever $\left(>38.5^{\circ} \mathrm{C}\right)$, ESR $>60 \mathrm{~mm}$ in the first hour and/or CRP $>3.0 \mathrm{mg} / \mathrm{dL}$ for low-risk populations, and fever $\left(>38^{\circ} \mathrm{C}\right)$, ESR $>30 \mathrm{~mm} / \mathrm{h}$ and/or CRP $>3.0 \mathrm{mg} / \mathrm{dLfor}$ moderate- and high-risk populations have been included as minor criteria for ARF.10

RHD is the well-recognized complication of ARF. In its fully established form, diagnosis of RHD is relatively straightforward. Traditionally, RHD was diagnosed by auscultation with a stethoscope of a heart murmur in those with a history of ARF. But, like diagnosis of rheumatic valvulitis by stethoscope alone, a significant proportion of milder cases of RHD may escape diagnosis, leading to underestimation of the 'true' prevalence of the disease, and failure of ensuring secondary prophylaxis earlier. As a diagnostic modality, echocardiography has proven to be more sensitive and specific than auscultation. ${ }^{13}$ RHD detected on echocardiography without an associated clinically pathological cardiac murmur is referred to as 'subclinical RHD'. With the introduction and wider availability of echocardiography, especially the Doppler echocardiography, these subclinical cases of RHD are being diagnosed, which are otherwise not apparent by auscultation only. Numerous papers published in recent years indicate that, the previously known prevalence of RHD actually represents the tip of the iceberg of the problem. Echo based 'true' prevalence of RHD is much higher than the previously known prevalence. ${ }^{14-20}$

The 2012 Australian guideline stressed that 'all patients with murmurs suggestive of valve disease, or a past history of RF, require echocardiography'. ${ }^{8}$ Concurrently, the WHF published new echo-based criteria for diagnosing RHD. Again the main objective was to pick up the subclinical cases so that the secondary prophylaxis can be instituted early to avoid further damage to the heart valves. The WHF echocardiographic criteria for $\mathrm{RHD}^{12}$ define 3 categories on the basis of assessment by 2D, continuous-wave, and color-Doppler echocardiography: 'definite RHD', 'borderline RHD', and 'normal'; 4 subcategories of 'definite RHD' and 3 subcategories of 'borderline RHD' exist, to reflect the various disease patterns. The morphological features of RHD and the criteria for pathological mitral and aortic regurgitation are also defined. The criteria are modified for those aged over 20 years.

Recent application of echocardiography in the diagnosis of ARF and RHD has brought about significant changes in the epidemiology of these diseases. However, the longterm significance of diagnosing these subclinical cases 
of rheumatic valvulitis in ARF and rheumatic carditis in RHD by a sensitive test like echocardiography is unknown at present.

At the advent of the new millennium, we are really unknown about our real situation. The recently published prevalence of ARF and RHD in Bangladesh, 0.9 per 1000 (ARF 0.6 per 1000 and RHD 0.3 per 1000) ${ }^{21}$, may also be an under-estimation of the 'real' prevalence. The existing Doctors' Manual on Rheumatic Fever ${ }^{22}$ is more than a decade old, so, it should be updated. Appropriate guidelines may be formulated in relation to RF and RHD to bring about uniformity and rationality in existing practice. In the changing context, echo-based, large-scale, preferably, nation-wide survey and clinical research should be conducted to determine the different aspects of ARF and RHD in Bangladesh. Certainly, the recently published guidelines, specially those by AHA and WHF should be considered. The information available thereby, would help to formulate national policy to combat this public health problem more efficiently in future. Otherwise, we will continue to feel a false sense of security and wellbeing of being in a better position in respect of $A R F$ and $R H D$.

\section{References:}

1. Remenyi B, Carapetis J, Wyber R, Taubert K, Mayosi BM; World Heart Federation. Position statement of the World Heart Federation on the prevention and control of rheumatic heart disease. Nat Rev Cardiol. 2013 May;10(5):284-92.

2. Tibazarwa KB, Volmink JA, Mayosi BM. Incidence of acute rheumatic fever in the world: a systematic review of population-based studies. Heart. 2008;94:1534-40.

3. Carapetis JR, Steer AC, Mulholland EK, Weber M. The global burden of group A streptococcal disease. Lancet Infect Dis. 2005; 5:685-94.

4. Carapetis JR. Rheumatic heart disease in Asia. Circulation. 2008;118:2748-53.

5. Jones $\mathrm{T}$. The diagnosis of rheumatic fever. JAMA. 1944;126(8):481-4.

6. Guidelines for the diagnosis of rheumatic fever. Jones Criteria, 1992 update. Special Writing Group of the Committee on Rheumatic Fever, Endocarditis, and Kawasaki Disease of the Council on Cardiovascular Disease in the Young of the American Heart Association. JAMA. 1992 Oct 21;268(15):2069-73.
7. Rheumatic fever and rheumatic heart disease: report of a WHO Expert Consultation, Geneva, 29 October-1 November 2001. Geneva: WHO, 2004.

8. RHDAustralia (ARF/RHD writing group), National Heart Foundation of Australia and the Cardiac Society of Australia and New Zealand. Australian guideline for prevention, diagnosis and management of acute rheumatic fever and rheumatic heart disease (2nd edition). 2012.

9. Atatoa-Carr P, Lennon D, Wilson N; New Zealand Rheumatic Fever Guidelines Writing Group. Rheumatic fever diagnosis, management, and secondary prevention: a New Zealand guideline. $N$ Z Med J. 2008 Apr 4;121(1271):59-69.

10. Gewitz MH, Baltimore RS, Tani LY, et al. American Heart Association Committee on Rheumatic Fever, Endocarditis, and Kawasaki Disease of the Council on Cardiovascular Disease in the Young. Revision of the jones criteria for the diagnosis of acute rheumatic Fever in the era of Doppler echocardiography: a scientific statement from the American Heart Association. Circulation. 2015 May 19;131(20):1806-18.

11. Tubridy-Clark M, Carapetis JR. Subclinical carditis in rheumatic fever: a systematic review. Int J Cardiol. 2007 Jun 25;119(1):54-8.

12. Reményi B, Wilson N, Steer A, et al. World Heart Federation criteria for echocardiographic diagnosis of rheumatic heart disease-an evidence-based guideline. Nat Rev Cardiol. 2012 Feb 28;9(5): 297-309.

13. Saxena A. Increasing detection of rheumatic heart disease with echocardiography. Expert Rev Med Devices. 2014 Sep;11(5):491-7.

14. Marijon E, Ou P, Celermajer DS, et al. Prevalence of rheumatic heart disease detected by echocardiographic screening. N Engl J Med. 2007 Aug 2;357(5):470-6.

15. Carapetis JR, Hardy M, Fakakovikaetau T, et al. Evaluation of a screening protocol using auscultation and portable echocardiography to detect asymptomatic rheumatic heart disease in Tongan schoolchildren. Nat Clin Pract Cardiovasc Med. 2008 Jul;5(7):411-7.

16. Paar JA, Berrios NM, Rose JD, et al. Prevalence of rheumatic heart disease in children and young 
adults in Nicaragua. Am J Cardiol. 2010 Jun 15;105(12):1809-14.

17. Bhaya M, Panwar S, Beniwal R, Panwar RB. High prevalence of rheumatic heart disease detected by echocardiography in school children. Echocardiography. 2010 Apr;27(4):448-53.

18. Saxena A, Ramakrishnan S, Roy A, et al. Prevalence and outcome of subclinical rheumatic heart disease in India: the RHEUMATIC (Rheumatic Heart Echo Utilisation and Monitoring Actuarial Trends in Indian Children) study. Heart. 2011 Dec;97(24):2018-22.

19. Baroux N, Rouchon B, Huon B, Germain A, Meunier JM, D'Ortenzio E. High prevalence of rheumatic heart disease in schoolchildren detected by echocardiography screening in New Caledonia. J Paediatr Child Health. 2013 Feb;49(2):109-14.

20. Rama Kumari N, Bhaskara Raju I, Patnaik AN, et al. Prevalence of rheumatic and congenital heart disease in school children of Andhra Pradesh, South India. J Cardiovasc Dis Res. 2013 Mar;4(1):11-4.

21. Zaman MM, Choudhury SR, Rahman S, Ahmed J. Prevalence of rheumatic fever and rheumatic heart disease in Bangladeshi children. Indian Heart J. 2015 Jan-Feb;67(1):45-9.

22. Pilot Project for Prevention and Control of Rheumatic Fever and Rheumatic Heart Diseases in Bangladesh. Doctors' Manual on Rheumatic Fever. Dhaka: Ministry of Health and Family Welfare and Japan International Cooperation Agency, 1988. 